\title{
Exploring the sociocultural aspect of narrative inquiry: A dynamic nursing research methodology
}

\author{
Esther Abena Adama*, Deborah Sundin, Sara Bayes \\ School of Nursing and Midwifery, Edith Cowan University, Western Australia
}

Received: April 10, 2016

Accepted: June 13, 2016

Online Published: July 15, 2016

DOI: $10.5430 /$ cns.v4n4p1

URL: http://dx.doi.org/10.5430/cns.v4n4p1

\begin{abstract}
Background: Human beings live storied lives and it is these stories that define the "self" in a community. Narrative inquiry, a qualitative research methodology seeks to understand social and cultural experiences of people and communities by studying stories from the perspective of individuals who are living the story and relating it to time, place and person. Narrative inquiry has two aspects: (1) the lived experiences (phenomenological aspect), and (2) the interactions of the stories with the story teller's sociocultural environment (ethnographical aspect). However, only the lived experiences aspect of narrative inquiry is overemphasized in the literature. The aspect that deals with the interactions of stories to the story teller's sociocultural environment has been overlooked.

Aim: To explore the sociocultural feature of narrative inquiry and to demonstrate how this methodology was used to explore both lived experiences and cultural aspects of parents' experiences of caring for preterm infants after discharge from four neonatal units in Ghana.

Conclusions: Given the dynamic nature of narrative inquiry, this article proposes that its use should not be limited to the study of experiences only. For a qualitative study that requires the study of both experiences and the interaction of participants with their sociocultural environment, narrative inquiry serves the purpose.
\end{abstract}

Key Words: Culture, Narrative inquiry, Parents, Preterm infants, Sociocultural

\section{INTRODUCTION}

\subsection{What is narrative inquiry?}

The term narrative inquiry was conceived by Connelly and Clandinin $^{[1]}$ as a research methodology in the educational research field. Its inception was inspired by Dewey ${ }^{[2]}$ assertion that "life is education". In other words, our experiences and education are intricately linked. Clandinin and Connelly ${ }^{[3]}$ placed more emphasis on the study of lived experiences using narrative inquiry. Thus, Connelly and Clandinin ${ }^{[4]}$ defined narrative inquiry as:

“... the study of experience as story... first and foremost a way of thinking about experience. Narrative inquiry as a methodology entails a view of the phenomenon. To use narrative inquiry methodology is to adopt a particular view of experience as phenomenon under study” (p. 479).

Narrative inquiry is concerned with the study of lived experiences using stories as data. ${ }^{[5,6]}$ Connelly and Clandinin ${ }^{[4]}$ viewed humans as individually and socially leading storied lives. Although Clandinin and Connelly ${ }^{[3]}$ emphasised that narrative inquiry studies the experiences of individuals, they also argued that this cannot be done in isolation without studying the socio-cultural factors that affect an experience. This was highlighted in the following statement "People are

\footnotetext{
* Correspondence: Esther Abena Adama, PhD Candidate; Email: estyadama@yahoo.com; Address: School of Nursing and Midwifery, Edith Cowan
} University, 270 Joondalup Drive, Perth WA 6027, Western Australia. 
individuals and need to be understood as such, but they cannot be understood only as individuals. They are always in relation, always in a social context." (p. 2). Through narrative inquiry an in-depth understanding of people's thoughts and values of an event may be gained. Narrative inquiry integrates time with culture to make meaning of an experience from the view of the storyteller. ${ }^{[7]}$ According to Riley and Hawe, ${ }^{[8]}$ narrative approach studies the sentences constructed by the storyteller closely for the meanings attached to them. Through narrative inquiry, life stories that are lived are re-told, reconstructed and re-lived. ${ }^{[9]}$

\subsection{What constitutes narrative inquiry methodology?}

The assumption underpinning this design is that people construct or give meanings to their experiences through storytelling. ${ }^{[8]}$ Clandinin $^{[10]}$ argued that human beings live by telling stories and it is these told stories that defines the "self" in a community and create meanings to the life of the "self". Narrative inquiry seeks to understand the social and cultural behaviour of a group of people, communities and contexts through the lived experiences of individuals. ${ }^{[5]}$ This means for a complete sense of the world to be made by the storyteller, the role of other people in the story should be made known. Gergen ${ }^{[8]}$ called these people the supporting cast. They have a role to play in the story; the manner in which the storyteller relates them to the story and the part they played in the story helps the storyteller to make meaning to the story. Therefore it is imperative to explore the effect of the cast on the stories in order to make complete sense of the stories.

Narrative inquiry combines various methodologies in its approach to research ${ }^{[11]}$ It combines the cultural characteristics of ethnography ${ }^{[12]}$ and the heuristic aspect of phenomenology. ${ }^{[10]}$ Emden $^{[13]}$ considered narrative inquiry to be one of the forms of the broader phenomenology in that it gives meaning to experiences. Based on Creswell, ${ }^{[14]}$ it is obvious that the dynamic nature of narrative inquiry has not been fully explored by researchers. Researchers such as ${ }^{[5,11,15]}$ have established for years that narrative inquiry is not only the study of an experience but also the socio-cultural way of living that affect the phenomenon under investigation. Most reported narrative inquiry studies are concerned with the study of lived experiences ${ }^{[1,10,16]}$ and largely neglecting the cultural factors that influence the phenomenon of the experiences under investigation. The cultural aspect of narrative inquiry cannot be distinguished from the stories of lived experiences. Stories are lived and narrated in a socio-cultural environment which has great influence on the experiences of an individual. This is well captured by Shacklock and Thorp ${ }^{[17]}$ when they wrote "Experiences and meaning making are encultured and co-constructed whether in daily life or through engaging in research". ${ }^{[17]}$ Moen ${ }^{[18]}$ put this in a simpler form as "...narratives therefore capture both the individual and the context” (p. 60).

\subsection{The three dimensional narrative inquiry space}

Clandinin and Connelly ${ }^{[3]}$ developed a framework for analysing narrative data. In this framework, stories of participants are analysed using the three dimensional spacetemporality, sociality and spatiality. These dimensions are incorporated in narrative analysis to give structure to the analysis of data obtained from narrative inquiry. In addition, re-storying, a process of rewriting stories to be analysed for key issues in a chronological sequence was also utilised in the initial stages of data analysis. ${ }^{[19]}$

Clandinin ${ }^{[10]}$ and Clandinin and Connelly ${ }^{[3]}$ described the three dimensional space as follows:

(1) Interaction/Sociality: shows the personal and social interactions that exist in the story

(2) Continuity/Temporality: explains the "time factor". That is, the story has a present, past and future

(3) Situation/Spatiality: shows the place in which the story took place

The socio-cultural context of a story is incorporated in the three dimensional space analysis framework of Clandinin and Connelly ${ }^{[3]}$ as sociality; however, they failed to explain the process of eliciting socio-cultural issues in narrative inquiry research. In this paper, we present narrative inquiry as a qualitative method and its effectiveness in exploring parents' lived experiences of caring for preterm infants after discharge from neonatal units, and we pay particular attention to the methodology's capacity for uncovering the cultural aspect of the phenomenon under investigation.

In the following paragraphs, we discussed how we used the narrative inquiry methodology to study both the experiences of parents of preterm infants in the community and the sociocultural factors that affect the phenomenon of caring for preterm infants in the absence of highly qualified medical practitioners. Data presented here form part of a larger study that explored the experiences of caring for preterm infants after discharge from neonatal from February to June, 2015. We used this data to demonstrate how we used narrative inquiry in our case to explore parent's experiences of caring for preterm infants and the socio-cultural factors that affect this experience in the community.

\section{METHODS}

\subsection{Setting}

The stories used in the demonstration of narrative inquiry were collected through face-to-face interview and partici- 
pants' observation at homes of parents after discharge from four neonatal units (one teaching hospital, three district hospitals) in Ashanti and Western region of Ghana in West Africa.

\subsection{Ethics and recruitment process}

Ethical approval from Edith Cowan University in Australia and Kwame Nkrumah University of Science and Technology was obtained before data collection. In addition, consent from hospital heads was obtained before accessing the neonatal units. Parents who were more than 18 years with preterm infants who have no other disabilities apart from being born preterm (gestational age less than 37 weeks) were approached and the study explained to them for their participation after they have been discharged. After discharge, parents who consented to be part of the study were contacted via mobile phones to book a meeting for data collection. At their homes, participants signed or thumb-printed a consent form before the face-to-face interviews.

\subsection{Methodology}

Qualitative research methodology narrative inquiry, was deemed appropriate for this study as we intended to explore both the lived experiences of caring for preterm infants and the socio-cultural factors affecting the care of preterm infants in the community. Initially, hermeneutic phenomenology was considered as we wanted to explore parent's experiences of caring for preterm infants. ${ }^{[20]}$ However, upon further examination, the authors were convinced that hermeneutic phenomenology could not completely cover the aim of the study which included exploring the socio-cultural factors influencing the care of the preterm infant in the community. We also noted that ethnography was insufficient as its focus is mainly on culture and social interactions among a group of people rather than on experience. Therefore, we settled on narrative inquiry which will give us the advantage of exploring both the experiences of parents and how their socio-cultural environment affect their experiences.

Application of narrative inquiry in exploring cultural aspect of parents' experiences of caring for preterm infants after discharge from neonatal unit

Data used for the demonstration of narrative inquiry were collected as part of a doctoral study that explored parent's experiences of caring for preterm infants after discharge in Ghana. Parents of preterm infants experience stressful situations during their hospitalisation in the neonatal units and after discharge. ${ }^{[21,22]}$ After discharge, Ghanaian parents rely on their social support networks to care for their preterm infants. Forty-two parents of preterm infants from one teaching hospital and three district hospitals in Ghana who were admitted at a neonatal unit were asked to tell their stories

Published by Sciedu Press about their care giving experiences at the hospital and after discharge. Stories were told of how parents experienced the care of the preterm infant in the neonatal unit and after discharge at home. Parents were visited at least three times at their homes to share their experiences.

As time, culture and people are considered important aspects in narrative inquiry, the relationship of the storyteller with the supporting cast must also be prioritized when analysing stories as these inform value placed on an experience. In our example with parent's experiences of caring for preterm infants in the community, ${ }^{[23]}$ we noted that individuals are closely linked to their culture and it is this culture that informs their experience. Therefore, it was virtually impossible to study our participants' experiences without studying the culture that defines their experiences. In situations like this, narrative inquiry presents as the best method for exploring the cultural perspective of individuals in order to have in-depth understanding of their experiences and to develop appropriate interventions.

Data collection involved four interviews over four months. Participants were recruited from four hospitals wherein neonatal services are provided in Ghana (three regional/district and one tertiary). Data collection, however, took place at the residences of the participants who consented to take part in the study. One of the researchers (EAA) and data collector for this project is herself a Ghanaian which enhanced the participants' comfort with the interview process. As the homes of the participants were considered their natural habitat, a very good interpersonal relationship was developed with the participants and their family prior to data collection. Culturally, Ghanaian family welcome people into their homes especially with the arrival of a newborn. However, as health visiting is not a usual practice in Ghana, participants were hesitant to allow the researcher, who they also knew to be a registered nurse into their homes during the first visit. It was made clear to participants that the researcher was not engaging with them in a nursing capacity. With this experience from two participants on the first visit, an initial familiarization visit was made to the rest of the participants' homes to create a rapport before the first set of face-to face interviews were organised. This made the parents feel more comfortable to tell their stories. Stories were collected until saturation was reached (172 interviews in total). Collecting data in participants' homes provided an opportunity to understand their ways of life and how they related to their social environment with the arrival of the new preterm infant. During the second, third and fourth home visits, participants were more relaxed and opened to tell more in-depth stories about their experiences both in the neonatal unit and home/community. In most cases, the 
participants were so engaged in the interview that they felt the researcher was part of their household, and hid nothing. This was achieved because of the researcher's deep appreciation of and non-judgemental approach towards participants' cultural practices.

In order to maximise engagement, we developed a semi structured interview guide that required parents to narrate their stories from the time of labour in the labour suite to the day of home discharge and thereafter. This structure was intended to include temporality which will allow parents to narrate their stories in a chronological manner, sociality which will demonstrate the interaction of parents with other individuals in caring for the preterm infant and spatiality which will show how the natural environment influence the care giving behaviour of parents. However, this was not achieved in all interviews as parents kept on going to and fro with their stories. Thus, during analysis, we used re-storying approach ${ }^{[19]}$ to write stories to flow in a chronological manner bearing in mind the interaction of parents with their socio-cultural environment and their interpretation of caring. Each interview with a mother, father and/or significant other lasted between 20 to 80 minutes.

As narrative inquiry is also concerned with the socio-cultural factors affecting the care of preterm infants, parents were asked to explain some of the cultural factors that affect the care of preterm infants in their local community. The participants' stories of their lived experiences follow a continuum from their experiences in the neonatal unit, through to taking their baby back to their home environment, and their relationship with the health workers and social support after discharge. Cultural practices that influenced the care of preterm infants after discharge were also explored. In order to understand the cultural practices of parents, additional data was taken from grandparents, aunties and members of the extended family who had joined the household to provide support for parents to care for their preterm infants.

The use of field notes and observation of parent's behaviour at their homes also provided rich data. Participant observations and field notes were used to complement interview data. Although narrative inquirers do not seek truth in one's experience, ${ }^{[24]}$ comparing our observations to interview responses from the participants revealed some degree of incongruence. A lot of tension was observed in what parents say, their behaviour and what they ought to be doing. For example, when one mother was interviewed at one week after discharge about how she maintained the nutrition of her preterm infant, she said, "I feed him with breast milk alone, no formula or water as told by the nurses" (Becca, Mother). However, five minutes later, an auntie came from the bedroom asking the same mother where she had kept the formula so that she can feed the baby because he was crying.

\section{NARRATIVE ANALYSIS OF DATA AND RE- SULTS}

Narrative inquiry goes beyond merely telling stories. ${ }^{[24]}$ Thus structured and in-depth analysis of the stories were done to explore the meaning attached to them. In our example, stories were analysed using the narrative analysis approach described by Riley and Hawe. ${ }^{[8]}$ Analysis of events, values, culture, norms, present stories and past events and their possible influence on parents' experiences were derived from the data. Analysis was based on how individuals experienced the phenomenon of caring for their preterm infants as events unfolded prior to neonatal unit admission, during the neonatal unit admission and at home after discharge. Analysis focused on how the individual's experiences changed as time, place and persons (healthcare workers to family and friends in the community) changed. As data was collected at various stages - one week, one month and four months after discharge it was analysed in a manner in which temporality, sociality and spatiality were evident. ${ }^{[3]}$ In order to understand how parent's experiences changed with time, the semi-structured interview questions remained the same throughout the various visits with slight changes to follow-up questions based on parents' responses to interview questions. This approach of data analysis provided a clear understanding of participants' experiences at various stages. That is at any stage, we were able to analyse how the stories related to the participant's present and past experience, their immediate social environment and cultural practices. Similar categories were matched and broader themes formed if the categories were similar.

In our example, experiences at neonatal units were analysed separately from those explored after discharge recognising the role of supporting casts in participants' stories. In the hospital, healthcare workers were the main cast while family and friends remained cast after discharge. Adama, Sundin ${ }^{[23]}$ showed how data was brought under three umbrella themes after neonatal unit discharge: (1) Grandmother's prescription, (2) Fighting for the preterm infant, and (3) Being in a confused state.

\section{Re-storying}

In narrative inquiry, analysis of data (mostly stories) is done by re-storying within the framework of the three dimensional narrative space framework. Ollerenshaw and Creswell ${ }^{[19]}$ defined re-storying "as the process of gathering stories, analyzing them for key elements of the story (e.g., time, place, plot, and scene), and then rewriting the story to place it within 
a chronological sequence" (p. 332).

In our data analysis, re-storying was employed to double check the recorded interviews. As some participants cannot read and write, the recorded interviews were given to one of the authors to listen and read the interpreted data. Stories were then re-told and confirmed where necessary with the participants. These were then arranged in chronological order to reflect temporality, sociality and spatiality in narrative inquiry. This is illustrated below in an interview with a mother of a 27-week-old preterm infant after discharge:

"You see here at home, I have all the time with my baby and daddy can hold him and feed him. I feel daddy is now part of his care than when we were in the hospital. The hospital was rather not accommodating for his (preterm infant) father. The feeding time is the same time fathers are allowed to visit, so you can imagine. No quality time. In the home, we have all the time and we can feed him for longer time. There's no rush to finish feeding within a certain time for the nurses to do their job." (Liz, mother)

This story was re-storied to cater for the times they happened, the casts involved and the space within which the stories occurred:

- Neonatal unit experience: "The hospital was rather not accommodating for his (preterm infant) father. The feeding time is the same time fathers are allowed to visit, so you can imagine. No quality time."

- Home experience: "I feel daddy is now part of his care than when we were in the hospital. In the home, we have all the time and we can feed him for longer time. There's no rush to finish feeding within a certain time for the nurses to do their job."

The example above shows how the story teller relates her experience with her husband (preterm infants' father), the nurses and her experiences at different times during her stay in the neonatal unit and after her discharge at home.

Data were then read over and over and grouped into similar categories and themes. This way, participants' experiences and their cultural practices were represented. This is illustrated in the following example:

(1) "I was also told by some family and friends that the baby was suffering from 'asram' She was stretching herself too much so they said it was 'asram' so my mother (grandmother) encouraged me to take her there and I did. I took her to the herbalist who treats 'asram' and he gave me some herbs to smear her body and by God's grace, she is doing well now." (Shu, mother)

(2) "My mother talked to one woman who is a herbalist about

Published by Sciedu Press my baby and she said I should bath him but I should apply this concoction called 'sheri' on him. It is a mixture of clay and some guava leaves herbs. I just apply it on him, his stomach, but head and face are not included so that he will grow bigger." (Lamisi, mother)

(3) “My mother said she didn't do anything when I was born (preterm). She just followed the advice of nurses and doctors and I survived so I should follow what they are saying and my baby will be fine. My mum said I was even smaller than this one (her baby)." (Duruwaa, mother)

(4) "My mum had this belief that babies who are just light weight and preterm babies are the cause of this traditional belief we have, we call 'asram' She was saying that the baby has asram so we should take her to the herbalist for them to give us drugs so that we'll come and do all that. We took her there when we were discharged from the hospital. So he (herbalist) gave us some herbs and said that when we come, we should soak and bath the baby with it." (Maabena, mother)

The above stories (1-4) carried one message-grandparents trying to influence the care of their preterm grandchildren. These findings were grouped and a theme - Grandmother's prescription was given. That is all the advice provided by the grandmothers of preterm infant regarding their care were captured under this theme. From the above stories, one can appreciate the cultural practice of having the grandmother around when the preterm infant is discharged home. The role of the grandmother cannot be underestimated with the arrival of a newborn. They are "home paediatricians" understanding the influence of the grandmother as a cultural phenomenon by the use of narrative inquiry revealed hidden cultural practices that may result in poor neonatal outcomes in the community.

By becoming aware of the cultural practices, our study identified some traditional diseases - "asram" and "esoro" - that were believed to have no cure in the hospital; they can only be cured by using alternative traditional means. A mother narrated her community's description of a child with "asram" as:

"Asram is a spiritual disease, they can give it to you when you are pregnant... they said when it comes to babies, their heads become longer and they continue to decrease in size... it is 'asram' which causes low birth weight and preterm birth." (Isha, mother)

Based on this believe, the community have special treatment for children with "asram" outside the hospital. The commonest form of treatment is the use of traditional herbs which have not been studied for their efficacy. A mother shared her 
experience as:

"My mum had this belief that babies who are just light weight and preterm babies are the cause of this traditional belief we have, we call 'asram' She was saying that the baby has asram so we should take her to the herbalist for them to give us drugs so that we'll come and do all that." (Maabena, mother)

In some cases, preterm infants were seen with herbs smeared on their body, bracelet made of chameleon and cowry shells during visit. This was supposed to prevent and cure the traditional disease "asram". To confirm this observations, parents were asked the significance of the herbs. By asking questions on cultural practices that affect the care of the preterm infants, specific data was obtained. The following interview illustrates this approach. This interview was conducted in the home of a mother (Pat) and her sister (Mary) who had relocated to Pat's residence to help with the care of her preterm twins:

Researcher: "How do your community members perceive the preterm twins?”

Pat: "They believe they are sick because of their smallish nature and they have started recommending some traditional medicines that will let them grow big."

Mary: "They see them to be sick but usually they advise us on how to properly care for them. They also encourage us to be mindful of their sick status and treat them with the best of care."

Researcher: "What sort of advice have family and friends been offering?"

Pat: "Some family members advise that I keep them warm, give them more breast milk, generally, they tell me to pay more attention to the babies."

Mary: "You see because their time was not due, they are very weak so as for me, I use some traditional medication to smear their bodies after bathing them. It is basically made of grounded guinea corn and shea butter mixed together. This will make them stronger. We took them to one woman who is a herbalist and she gave us this mixture. As soon as we finish bathing them, we smear this on their body to strengthen their muscles and bones so that they can become stronger."

Researcher: "What else do you do culturally for the babies who were born preterm?"

Mary: "For now this is the only thing we do for them but if they were weaker than this, we could also put grounded beans and some concoctions on their chest. This will help to fill all the holes in the heart. This is done all the time, but because these ones are a bit stronger, we don't add the beans.”

The interview above shows how the community perceive preterm infants and some practices in the community. The process of eliciting responses on cultural practices was always related to the way things are done in the community. The role of the traditional herbalist is very significant in the treatment of childhood disease including preterm birth. The manner in which the interview questions were designed and responses analysed have highlighted the cultural practices of caring for the preterm infant in the community and how parent's experience this care in the community.

In another interview with a mother (Maabena) and father (Kojo) of a preterm infant, this was disclosed:

Researcher: "What are some of the cultural practices that influence the care of the preterm infant in your community?'

Maabena: "Usually, the baby is kept in the room for a week from sunlight, as they are still growing so if you expose them to sunlight before one week, they may be sick. We also give enemas using guava leaves, bark of some plants and special grass. For smaller babies, they are given this enema so that they will not get constipated and grow lean. If you don't do the enema, they cannot grow well."

Reseacher: "What is the frequency of the enema?"

Maabena: "It is done on every other day or every three days."

Researcher: "Anything else?"

Kojo: "I think there is another practice where the baby is kept in a big calabash but that one is in the villages. The calabash protects the preterm infant from cold. It keeps them warm. I have seen it once in the village."

As narrative inquirers allow stories to be told from the perspective of the storyteller, participants were encouraged to tell their stories with regard to the cultural practices whether or not they practiced them. In the above interview, this community believes in keeping the preterm infant healthy. Constipation in an infant was perceived as the reason for being light weight. Therefore, parents and their social support networks will endeavour to maintain the preterm infant constipation free by using every means including herbal enemas with questionable efficacy. Similar interview with another mother (Isha) is recorded below:

Researcher: "What are some of the cultural practices that influence the care of the preterm infant in your community?"

Isha: "Not really, there's no cultural practice I know of, but I only keep my baby indoors. I will keep him indoors or cover him with a big cloth anytime I am going out so that the bad 
eyes will not see them.”

Researcher: "What do you mean by bad eyes?"

Isha: "They are the ones that give the babies 'asram' They are the bad people that go about giving children 'asram' so that If you don't hide your baby, they can easily attack her with asram. For such babies [preterm infants], they are not supposed to come out at all until they are able to withstand the asram disease. If you want to bring your baby out without fear of asram, then you have to go and get some herbal protection for the baby. Yes, many people do it. I will do it at the appropriate time but for now, he will stay indoors, windows and doors closed to protect him."

During this interview, the interviewer learnt to be nonjudgemental in order to engage participants. The stories of cultural practices are very important in public health interventions, yet they are most often neglected by researchers. Narrative inquiry provides the platform to elicit responses on both experiences and cultural practices. Most participants demonstrated their cultural practices in a manner that appeared as if they were not given any pre-discharge education in hospitals. This shows that people are more likely to practice their culture more comfortably than what was taught at the hospital prior to their discharge. Culturally, participants believe there are "bad eyes" that require specific protection other than the hospital treatment. These "bad eyes" are believed to cause other forms of diseases. Thus seeking traditional remedy was the best way to protect one's child. This believe informed their practice of keeping their babies indoors for a certain period of time.

The use of narrative inquiry to explore cultural factors influencing a phenomenon is not merely based on data collection, but also on the structured analysis of the data in the light of the three dimensional narrative space. This approach provides an in-depth understanding of the cultural principles in a given population, thus helping to make health workers more culturally aware and to provide culturally sensitive care. In our study, narrative inquiry as a research method has provided evidence on the lived experiences of participants and the cultural practices that influence these experiences of caring for their preterm infants post-discharge. This way, we are able to understand why people behave the way they do and also support them to make good health decisions. Understanding people's way of life provides the foundation for planning services to meet their needs.

\section{Conclusions}

Narrative inquiry is a dynamic qualitative research method that has been used widely in education, health and social science research. It is acknowledged that narrative inquiry studies the lived experiences and the cultural and/or social factors influencing the phenomenon under study. Researchers use stories as data to explore the meaning individuals ascribe to their experiences. As a method that has an advantage of eliciting two very important aspects of qualitative methodologieslived experiences (heumenstic phenomenology) and cultural influence (ethnographic), it is important to explore the dynamic power of narrative inquiry in studying the influences of socio-cultural factors in the experiences of individuals.

To demonstrate the unique feature of narrative inquiry in eliciting both the experiences and the socio-cultural factors affecting parents' lived experiences of caring for preterm infants after neonatal unit discharge in the Ghanaian community, we have shown how data was collected, analysed and reported using narrative inquiry. Socio-cultural revelations from this project can form the basis of health interventions for discharged preterm infants in the Ghanaian community.

\section{CONFlicts OF INTEREST Disclosure}

No conflict of interest has been declared by the authors.

\section{REFERENCES}

[1] Connelly FM, Clandinin DJ. Stories of experience and narrative inquiry. Educational Researcher. 1990; 19(5): 2-14. http://dx.doi . org/10.3102/0013189X019005002

[2] Dewey J. Experience and education. New York: Simon \& Schuster; 1938.

[3] Clandinin DJ, Connelly FM. Narrative inquiry: Experience and story in qualitative research. San Francisco: Jossey-Bass; 2000.

[4] Connelly FM, Clandinin DJ. Narrative inquiry. In: J. L. Green, G. Camilli, P. Elmore, editors. Handbook of complementary methods in education research. $3^{r d}$ ed. Mahwah, NJ: Lawrence Erlbaum; 2006. $477-87 \mathrm{p}$.

Published by Sciedu Press
[5] Bleakley A. Stories as data, data as stories: making sense of narrative inquiry in clinical education. Medical education. 2005; 39(5): 534-40. PMid: 15842721. http://dx.doi.org/10.1111/j.1365-2929. 2005.02126. $\mathrm{x}$

[6] Baughman KR, Aultman JM, Ludwick R, et al. Narrative analysis of the ethics in providing advance care planning. Nursing Ethics. 2014; 21(1): 53-63. PMid: 23702895. http://dx.doi.org/10.1177/0 969733013486795

[7] Simms K. Paul Ricoeur. London: Psychology Press; 2003. http: $/ / \mathrm{dx}$.doi.org/10.4324/9780203165508

[8] Riley T, Hawe P. Researching practice: the methodological case for narrative inquiry. Health Education Research. 2005; 20(2): 226-36. PMid: 15479707. http://dx.doi.org/10.1093/her/cyg122 
[9] Clandinin DJ, Connelly FM. Personal experience methods. Handbook of qualitative research. 1994; 413: 427.

[10] Clandinin DJ. Narrative inquiry: A methodology for studying lived experience. Research Studies in Music Education. 2006; 27(1): 44-54. http://dx.doi.org/10.1177/1321103X060270010301

[11] McAllister MM. In harm's way: a postmodern narrative inquiry. Journal of Psychiatric and Mental Health Nursing. 2001; 8(5): 391-7. PMid: 11882158. http://dx.doi.org/10.1046/j.1365-2850. 2001.00408. $\mathrm{x}$

[12] Creswell JW. Qualitative inquiry and research design: Choosing among five approaches: Sage; 2012.

[13] Emden C. Conducting a narrative anlysis. Collegian. 1998; 5(3): 34-9. http://dx.doi.org/10.1016/S1322-7696(08)60299-1

[14] Creswell JW. Research Design: Qualitative, Quantitative, and Mixed Method. Manual Therapy. 2009; 16(1): 223-223.

[15] Clandinin DJ, Rosiek J, Clandinin D. Mapping a landscape of narrative inquiry. Handbook of narrative inquiry: Mapping a methodology. 2007: 35-75. http://dx. doi .org/10.4135/9781452226552

[16] Elliott J. Using narrative in social research: Qualitative and quantitative approaches: Sage; 2005. http://dx.doi.org/10.4135/978 0857020246

[17] Shacklock G, Thorp L. Life history and narrative approaches. Research methods in the social sciences. 2005: 156-63.
[18] Moen T. Reflections on the narrative research approach. International Journal of Qualitative Methods. 2008; 5(4): 56-69.

[19] Ollerenshaw JA, Creswell JW. Narrative research: A comparison of two restorying data analysis approaches. Qualitative Inquiry. 2002; 8(3): 329-47. http://dx.doi.org/10.1177/107780040 08003008

[20] Annells M. Hermeneutic phenomenology: Philosophical perspectives and current use in nursing research. Journal of advanced nursing 1996; 23(4): 705-13. PMid: 8675887. http://dx.doi.org/10. 1111/j.1365-2648.1996.tb00041.x

[21] Aagaard H, Hall EO. Mothers' experiences of having a preterm infant in the neonatal care unit: a meta-synthesis. Journal of Pediatric Nursing. 2008; 23(3): e26-e36. PMid: 18492543. http: //dx.doi.org/10.1016/j.pedn.2007.02.003

[22] Auslander GK, Netzer D, Arad I. Parental Anxiety Following Discharge From Hospital of Their Very Low Birth Weight Infants. Family Relations. 2003; 52(1): 12-21. http://dx.doi.org/10.1111/j $.1741-3729.2003 .00012 . \mathrm{x}$

[23] Adama EA, Sundin D, Bayes S. Grandmothers of preterm infants, blessing or curse? Parents' experiences of caring for discharged preterm infants. [Journal article manuscript]. In press 2016.

[24] Duff PA, Bell JS. Narrative research in TESOL: Narrative inquiry: More than just telling stories. TESOL quarterly. 2002; 36(2): 207-13. http://dx.doi.org/10.2307/3588331 